\title{
Chlamydia trachomatis during pregnancy and childhood asthma-related morbidity: a population-based prospective cohort
}

\author{
Evelien R. van Meel ${ }^{1,2}$, Marina Attanasi ${ }^{1,2}$, Vincent W.V. Jaddoe ${ }^{1,3}$, \\ Irwin K.M. Reiss ${ }^{4}$, Henriëtte A. Moll ${ }^{3}$, Johan C. de Jongste ${ }^{2}$ and Liesbeth Duijts $\circledast^{2,4}$ \\ Affiliations: ${ }^{1}$ The Generation R Study Group, Erasmus MC, University Medical Center Rotterdam, Rotterdam, \\ The Netherlands. ${ }^{2}$ Dept of Pediatrics, Division of Respiratory Medicine and Allergology, Erasmus MC, \\ University Medical Center Rotterdam, Rotterdam, The Netherlands. ${ }^{3}$ Dept of Pediatrics, Erasmus MC \\ University Medical Center Rotterdam, Rotterdam, The Netherlands. ${ }^{4}$ Dept of Pediatrics, Division of \\ Neonatology, Erasmus MC, University Medical Center Rotterdam, Rotterdam, The Netherlands.
}

Correspondence: Liesbeth Duijts, Erasmus MC, University Medical Center Rotterdam, Sp-3435, P0 Box 2060 , 3000 CB Rotterdam, The Netherlands. E-mail: l.duijtsderasmusmc.nl

@ERSpublications

Chlamydia trachomatis infection during pregnancy is associated with increased odds of wheezing and asthma, and impaired lung function in childhood, and may be a target for prevention strategies focused on improving offspring respiratory health https://bit.ly/34fSXda

Cite this article as: van Meel ER, Attanasi M, Jaddoe VWV, et al. Chlamydia trachomatis during pregnancy and childhood asthma-related morbidity: a population-based prospective cohort. Eur Respir J 2020; 56: 1901829 [https://doi.org/10.1183/13993003.01829-2019].

\section{ABSTRACT}

Introduction: Chlamydia trachomatis is the most commonly reported sexually transmitted disease and although infection during pregnancy is associated with neonatal complications, long-term respiratory consequences are unknown. We aimed to determine whether C. trachomatis infection during pregnancy is associated with asthma-related symptoms across childhood

Methods: This study among 2475 children and their mothers was embedded in a population-based prospective cohort study. Maternal urine samples were tested for C. trachomatis infection during pregnancy. Questionnaires provided information on childhood physician-attended lower respiratory tract infections and wheezing, and current asthma at age 10 years. Lung function was measured by spirometry at age 10 years.

Results: The prevalence of C. trachomatis infection during pregnancy was $3.2 \%$ (78 out of 2475). C. trachomatis infection during pregnancy was not associated with lower respiratory tract infections until age 6 years, but was associated with a higher odds of wheezing in children until age 10 years (OR 1.50 (95\% CI 1.10-2.03)). C. trachomatis infection during pregnancy was associated with an increased odds of asthma (OR 2.29 (95\% CI 1.02-5.13)), and with a lower forced expiratory volume in $1 \mathrm{~s} /$ forced vital capacity and forced expiratory flow at $75 \%$ of forced vital capacity (z-score difference -0.28 (95\% CI $-0.52-0.04)$ and -0.24 (95\% CI $-0.46--0.01)$, respectively) in children at age 10 years. The observed associations were only partly explained by mode of delivery, gestational age at birth or birthweight.

Conclusions: C. trachomatis infection during pregnancy is associated with increased odds of wheezing, asthma and impaired lung function. The causality of the observed associations and potential underlying mechanisms need to be explored. 


\section{Introduction}

Chlamydia trachomatis is the most commonly reported sexually transmitted disease in Western countries [1-3]. It is hypothesised that C. trachomatis during pregnancy could affect childhood respiratory health through direct or indirect effects. C. trachomatis during pregnancy could directly lead to placental inflammation, potentially leading to altered immune and atopic development, and persistent risks of respiratory morbidity of the child $[4,5]$. C. trachomatis infection during pregnancy is known to be associated with a 1.3- to 4-fold increased risk of neonatal complications such as perinatal mortality, pre-term birth, low birthweight and pneumonia [4, 6-10]. Pre-term birth, low birthweight and neonatal respiratory disease are known to predispose to respiratory morbidity at later ages [11-13]. Therefore, C. trachomatis during pregnancy might also be associated with respiratory health in later life indirectly through these altered birth characteristics. Previous small-sample-sized observational studies focused mostly on the association of $C$. trachomatis infection in children with their respiratory health and suggested that an infection in infancy is associated with adverse respiratory health [14, 15]. Only one prospective cohort study was performed, studying the association between $C$. trachomatis and asthma in a subanalysis only, and reported that treatment for C. trachomatis during pregnancy was associated with a 3 -fold increased risk of asthma in the offspring at age 7 years [16]. However, no studies have examined the association of $C$. trachomatis during pregnancy with respiratory health or with objective lung function measurements later in childhood.

We hypothesise that $C$. trachomatis during pregnancy leads to fetal respiratory and immunological developmental adaptations, predisposing children to long-term asthma-related morbidity. We examined in a population-based prospective cohort study the associations of C. trachomatis infection during pregnancy with the odds of lower respiratory tract infections, wheezing, asthma and impaired lung function in children. We also examined whether any associations were explained by mode of delivery, gestational age at birth or birthweight.

\section{Methods \\ Design}

This study was embedded in the Generation R Study, a population-based prospective cohort from early fetal life onwards in Rotterdam in the Netherlands, comprising 9901 children and their mothers [17]. The study has been approved by the Medical Ethical Committee of the Erasmus MC (University Medical Center Rotterdam, Rotterdam, The Netherlands; MEC-2012-165). Written informed consent was obtained from mothers, parents or legal representatives of all children. The C. trachomatis substudy was performed between February 2003 and January 2005, and comprised 4055 mothers and children [6]. Non-singleton or non-live-born children and children without information on at least one of the respiratory outcomes were excluded, which left a total of 2475 children and their mothers for the current analysis. The flowchart of participants included for analysis is given in figure 1 .

\section{C. trachomatis infection during pregnancy}

Women provided a first-void urine specimen to test for C. trachomatis at enrolment, as described previously [6]. In summary, urine samples were stored at $4^{\circ} \mathrm{C}$, transported the same or following working day and processed within $24 \mathrm{~h}$ of receipt by the laboratory. DNA was isolated from five pooled urine specimens using the MagNA Pure LC Bacterial DNA Isolation Kit III (Roche Molecular Systems, Alameda, CA, USA) and amplified by PCR (Cobas Amplicor, Roche Molecular Diagnostics, Branchburg, NJ, USA). Urine samples from positive pools were individually re-tested and reported as negative or positive. Due to the observational nature of this study and the fact that screening for C. trachomatis during pregnancy was not part of standard care, results were not reported back to the women.

\section{Asthma and asthma-related morbidity in childhood}

We obtained information on lower respiratory tract infections (physician-attended pneumonia, bronchitis or bronchiolitis) by annual questionnaires from birth to age 4 years and at age 6 years. Information on wheezing was obtained at similar ages and at age 10 years. Information on asthma medication use in the past 12 months was obtained during the visit at the research centre (median age 9.8 years; 5-95\% range 9.510.4 years). Asthma was defined as ever-diagnosis of asthma, obtained by questionnaire at age 10 years, with either wheezing or medication use in the past 12 months. All questions on wheezing and asthma were based on the International Study on Asthma and Allergy in Childhood (ISAAC) questionnaire [18].

We performed spirometry according to the American Thoracic Society (ATS)/European Respiratory Society (ERS) recommendations during the visit at the research centre at age 10 years. Children $(n=154)$ with a $>5 \%$ deviation in forced expiratory volume in $1 \mathrm{~s}\left(\mathrm{FEV}_{1}\right)$ or forced vital capacity (FVC) but with at least one blow with adequate reach and duration of plateau according to ATS/ERS criteria were additionally included $[19,20]$. Lung function measures included $\mathrm{FEV}_{1}, \mathrm{FVC}, \mathrm{FEV}_{1} / \mathrm{FVC}$ and forced 


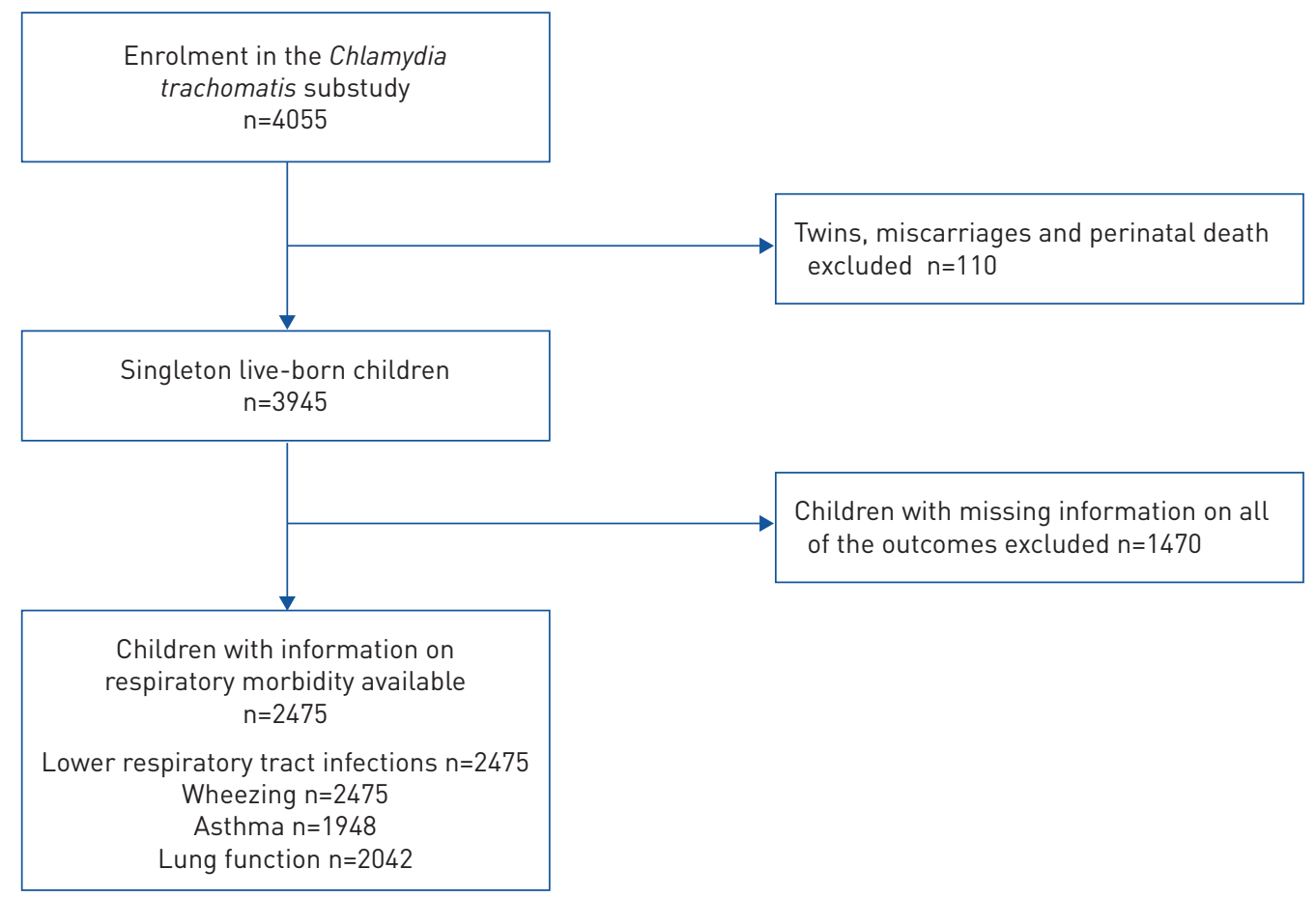

FIGURE 1 Flowchart of participants included for analysis.

expiratory flow at $75 \%$ of FVC $\left(\mathrm{FEF}_{75 \%}\right)$, and were converted into sex-, height-, age- and ethnicity-adjusted $\mathrm{z}$-scores according to the Global Lung Initiative reference data [21].

\section{Covariates}

Information on maternal age, educational level, risk behaviour including ever-treatment for sexually transmitted diseases and multiple sexual partners in the year before pregnancy, psychiatric symptoms, smoking during pregnancy, and alcohol use during pregnancy was obtained by multiple questionnaires during pregnancy [17]. Midwife and hospital registries provided information on mode of delivery, child's sex, gestational age at birth and birthweight [17]. Child's ethnicity was based on questionnaires during pregnancy. Questionnaires after birth provided information about child's breastfeeding and day care attendance.

\section{Statistical analysis}

First, we performed a loss-to-follow-up analysis by comparing characteristics of children included in our study and those lost to follow-up by using independent samples t-tests, Mann-Whitney $U$-tests and Pearson's Chi-squared tests. Second, we used generalised estimating equation models to examine the associations of $C$. trachomatis infection during pregnancy with the odds of lower respiratory tract infections and wheezing of the child until age 6 and 10 years, respectively. These models take the correlations between repeated measurements of either lower respiratory tract infections or wheezing within the same subject into account. Both an unstructured and first-order autoregressive correlation structure was tested, and the difference between these matrices was assessed by means of the quasilikelihood under the independence model criterion (QIC) statistic [22, 23]. Given that both correlation structures yielded similar results, but with a lower QIC for the first-order autoregressive correlation structure, this structure was used in the final model. Third, we used logistic and linear regression models to examine the associations of $C$. trachomatis infection during pregnancy with the odds of asthma and lung function measures, respectively. All multivariable analyses were adjusted for maternal age, educational level, psychiatric symptoms, and smoking and alcohol use during pregnancy, and child's ethnicity, breastfeeding and day care attendance in the first year of life (confounder model). Confounders were selected from literature first, and were subsequently tested for their association with both the determinant and the outcome, or a change of the unadjusted effect estimates of $\geqslant 10 \%$ when added to the univariate model [2428]. A directed acyclic graph was created to visualise the relationship between exposure, outcome and possible covariates (supplementary figure S1). Confounders were included in the final model if they were either associated with determinant and outcome, and not in the causal pathway, or if the effect estimate changed by $\geqslant 10 \%$ when they were included. To address potential residual confounding related to $C$. 
trachomatis infection during pregnancy, we additionally examined whether risk behaviour including ever-treatment for a sexually transmitted disease (yes/no) and multiple sexual partners in the year before pregnancy (yes/no) differed among women with and without $C$. trachomatis during pregnancy by means of Fisher's exact test.

We examined if the associations of $C$. trachomatis during pregnancy with respiratory morbidity were explained by mode of delivery, gestational age or birthweight by additionally adjusting for these variables (full model). We assessed the change in effect estimates after additional adjustment for these variables, by using the following formulas for percentage change:

$$
100 \times\left(\text { Effect }_{\text {estimate }} \text { full model }- \text { Effect estimate }_{\text {original model }}\right) /\left(\text { Effect }_{\text {estimate }} \text { original model }-1\right)
$$

for categorical lower respiratory tract infections, wheezing and asthma, and

$$
100 \times\left(\text { Effect estimate }_{\text {full model }}-\text { Effect estimate }_{\text {original model }}\right) /\left(\text { Effect }_{\text {estimate }} \text { original model }\right)
$$

for continuous lung function measures [11]. Additionally, we tested possible effect modification by gestational age at urine sampling for Chlamydia diagnosis by adding an interaction term to the model. Missing data in covariates, and in repeated measures of lower respiratory tract infections and wheezing, were imputed by the multiple imputation method using chained equations to select the most likely value for a missing response [29], creating 10 new datasets. Since we observed no major differences in the magnitude or direction of the effect estimates between analyses with imputed missing data and complete cases only, we only present the results based on imputed datasets. All measures of association are presented as odds ratios or z-score differences and their corresponding 95\% confidence intervals. Statistical analyses were performed using SPSS version 24.0 for Windows (IBM, Armonk, NY, USA), SAS version 9.4 for Windows (SAS Institute, Cary, NC, USA) and R version 3.4.1 (https://cran.r-project.org).

\section{Results}

\section{Subject characteristics}

Maternal and child characteristics are presented in table 1. The prevalence of $C$. trachomatis infection during pregnancy was $3.2 \%$ (78 out of 2475). The prevalence of childhood lower respiratory tract infections decreased after age 2 years, while the prevalence of childhood wheezing decreased gradually until age 10 years. Among the children born to mothers without versus with $C$. trachomatis during pregnancy, the prevalence of asthma at age 10 years was 5.3\% (101 out of 1893) versus $14.5 \%$ (eight out of 55) ( $\left.\mathrm{p}_{\text {difference }}=0.004\right)$. Main results of the loss-to-follow-up analysis showed that children not included in the study had a mother who more often had C. trachomatis during pregnancy, and had a lower gestational age at birth and birthweight (supplementary table S1).

\section{C. trachomatis, asthma and asthma-related morbidity}

None of the children with a lower respiratory tract infection at age 6 months were born to a mother with C. trachomatis infection during pregnancy and therefore only lower respiratory tract infections from age 1 year onwards were included in the model. C. trachomatis infection during pregnancy was not associated with annual or overall odds of lower respiratory tract infections in childhood (figure 2). Compared with children born to mothers without $C$. trachomatis infection during pregnancy, those born to mothers with C. trachomatis infection during pregnancy had an increased odds of wheezing until age 10 years (OR 1.50 (95\% CI 1.10-2.03)) (figure 2) and an increased odds of asthma (OR 2.29 (95\% CI 1.02-5.13)) (table 2). Additionally, these children had a lower $\mathrm{FEV}_{1} / \mathrm{FVC}$ (z-score difference -0.28 (95\% CI $\left.-0.52--0.04\right)$ ) and $\mathrm{FEF}_{75 \%}$ (z-score difference $-0.24(95 \% \mathrm{CI}-0.46--0.01)$ ) at age 10 years, but not a lower $\mathrm{FEV}_{1}$ or FVC (table 2). The proportion of women ever treated for a sexually transmitted disease did not differ between the groups with and without $C$. trachomatis infection during pregnancy $(12.1 \%$ versus $11.6 \% ; \mathrm{p}=0.84)$. The proportion of women having multiple sexual partners in the year before pregnancy in the group with C. trachomatis during pregnancy was lower than in the group without $(0 \%$ versus $7.6 \%$; $=0.02)$.

Additional adjustment for mode of delivery, gestational age at birth or birthweight did not materially change the effect estimates of the association of C. trachomatis infection during pregnancy with childhood respiratory health (table 2 for asthma and lung function, and supplementary tables S2 and S3 for lower respiratory tract infections and wheezing, respectively). The percentages change in the association of C. trachomatis during pregnancy with lung function and asthma after additional adjustment for birth characteristics ranged from $-6.2 \%$ to $1.1 \%$ (table 2 ) and were nonsignificant. The percentage changes for the overall odds of lower respiratory tract infection and wheezing were $-2.7 \%(95 \% \mathrm{CI}-156-175 \%)$ and $-5.0 \%$ (95\% CI $-24.7--0.2 \%)$, respectively. There was no effect modification by gestational age at diagnosis for associations of $C$. trachomatis with respiratory health $\left(p_{\text {interaction }}=0.434-0.887\right)$. 
TABLE 1 Characteristics of children and their mothers

\section{Subjects}

\section{Maternal characteristics}

Age years

Caesarean section

Low/middle education level

Maternal psychiatric symptoms

Smoking during pregnancy

Alcohol use during pregnancy

Chlamydia trachomatis infection during pregnancy

\section{Child's characteristics}

Female sex

Gestational age at birth weeks

Birthweight $g$

European ethnicity

Ever breastfeeding

Day care attendance first year

Lower respiratory tract infections

Age 1 year

Age 2 years

Age 3 years

Age 4 years

Age 6 years

Wheezing

Age 1 year

Age 2 years

Age 3 years

Age 4 years

Age 6 years

Age 10 years

Asthma\#

Lung function measures ${ }^{\#}$

$\mathrm{FEV}_{1} \mathrm{~L}$

FVC L

$\mathrm{FEV}_{1} / \mathrm{FVC} \%$

FEF $_{75 \%} \mathrm{~L} \cdot \mathrm{S}^{-1}$
Full group

Chlamydia-negative

2397

$30.86 \pm 4.7$
$309(12.9)$
$1161(48.4)$
$.15(0.06-0.31)$
$589(24.6)$
$1373(57.3)$
NA

1193 (49.8)

1230 (49.7)

40.1 (39.3-41.0)

$3446 \pm 546$

1556 (62.9)

2301 (93.0)

1350 (54.5)

210 (8.5)

317 (12.8)

203 (8.2)

159 (6.4)

145 (5.8)

773 (31.2)

541 (21.9)

377 (15.2)

381 (15.4)

294 (11.9)

$162(6.5)$

109 (5.6)

$2.02(0.30)$

$2.34(0.37)$

86.94 (5.68)

$1.16(0.35)$
40.1 (39.3-41.0)

$3450 \pm 545$

1591 (67.3)

2229 (93.0)

1325 (55.3)

202 (8.4)

310 (12.9)

$193(8.1)$

149 (6.2)

139 (5.8)

741 (30.9)

519 (21.7)

354 (14.8)

363 (15.1)

281 (11.7)

152 (6.3)

101 (5.3)

$2.03(0.30)$

$2.34(0.37)$

$86.9(5.7)$

$1.16(0.35)$
Chlamydia-positive

78

$27.58 \pm 6.2$

$12(15.4)$

56 (71.8)

$0.27(0.12-0.45)$

$23(29.5)$

$44(56.4)$

NA

37 (47.4)

40.0 (39.1-41.0)

$3327 \pm 627$

30 (41.0)

71 (91.0)

25 (32.1)

8 (10.2)

7 (9.0)

10 (12.8)

10 (12.8)

6 (7.6)

32 (41.0)

22 (28.2)

23 (29.5)

18 (23.1)

13 (16.7)

11 (14.1)

8 (14.5)

$1.97(0.32)$

$2.27(0.39)$

85.9 (5.1)

$1.04(0.32)$

Data are presented as $\mathrm{n}$, mean $\pm \mathrm{SD}, \mathrm{n}(\%)$ or median (interquartile range). NA: not applicable; $\mathrm{FEV}_{1}$ : forced expiratory volume in $1 \mathrm{~s}$; FVC: forced vital capacity; $\mathrm{FEF}_{75 \%}$ : forced expiratory flow at $75 \%$ of $\mathrm{FVC}$. \#: data were missing and not imputed for asthma (n=527) and lung function measures $(\mathrm{n}=433)$.

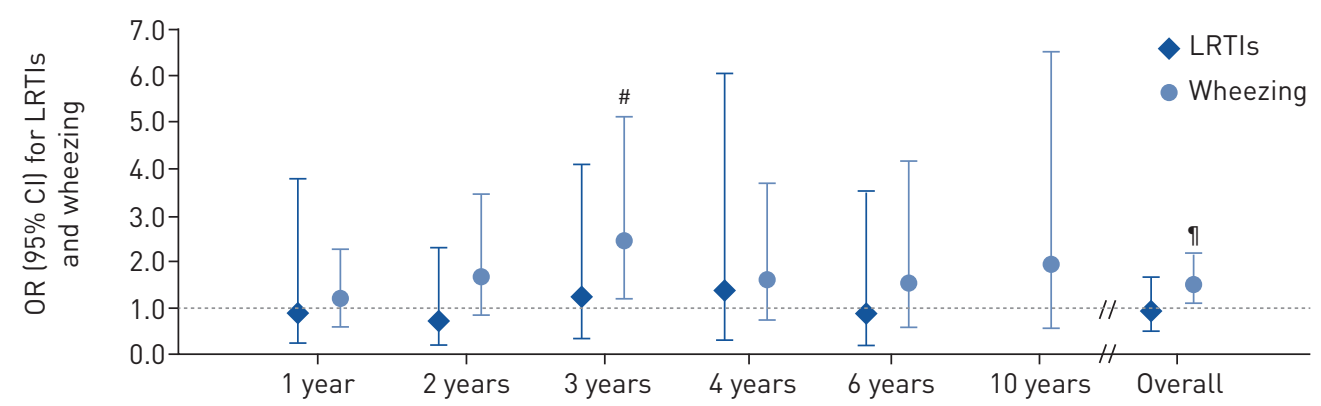

FIGURE 2 Associations of Chlamydia trachomatis infection during pregnancy with lower respiratory tract infections (LRTIs) from age 1 to 6 years and wheezing from age 1 to 10 years. Odds ratios with $95 \%$ confidence intervals from generalised estimating equation models. Models are adjusted for maternal age, educational level, psychiatric symptoms, and smoking and alcohol use during pregnancy, and child's ethnicity, breastfeeding and day care attendance in the first year of life. ${ }^{\#}: p=0.028 ;{ }^{\uparrow}: p=0.013$. 
TABLE 2 Associations of Chlamydia trachomatis infection during pregnancy with lung function and asthma

n FEV 1 z-score FVC z-score $\quad$ FEV $_{\mathbf{1}} / \mathrm{FVC}$ z-score $\quad$ FEF $_{\mathbf{7 5} \%}$ z-score difference $(95 \% \mathrm{CI})$ difference $(95 \% \mathrm{CI})$ difference $(95 \% \mathrm{CI})$ difference $(95 \% \mathrm{CI})$
Asthma

OR $(95 \% \mathrm{CI})$

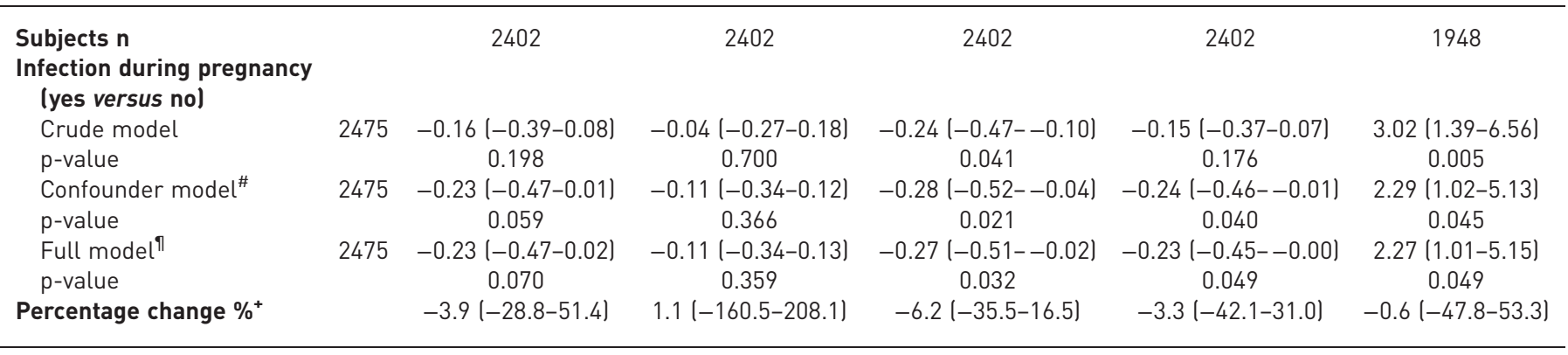

Data are presented are changes in z-score or odds ratio, derived from linear and logistic regression models, respectively. FEV $\mathrm{F}_{1}$ forced expiratory volume in $1 \mathrm{~s}$; FVC: forced vital capacity; $\mathrm{FEF}_{75 \%}$ : forced expiratory flow at $75 \%$ of FVC. \#: adjusted for maternal age, educational level, psychiatric symptoms, and smoking and alcohol use during pregnancy, and child's ethnicity, breastfeeding and day care attendance in the first year of life; ${ }^{\text {ๆ: }}$ additionally adjusted for mode of delivery, gestational age at birth and birthweight; ${ }^{+}$: percentage change $(95 \% \mathrm{CI})$ is between the full model and the confounder model.

\section{Discussion}

We observed that children of mothers with a C. trachomatis infection during pregnancy had increased odds of wheezing, asthma and impaired lung function in childhood. These observed associations with asthma and lung function were not explained by mode of delivery, gestational age at birth or birthweight, while the associations with overall wheezing were partly explained by these birth characteristics. C. trachomatis infection during pregnancy was not associated with lower respiratory tract infections across childhood.

\section{Strengths and limitations}

The major strength of this study is that it is embedded in a large prospective population-based cohort with longitudinal measurement of multiple respiratory outcomes and adjustment for relevant confounders. Additionally, we used a highly sensitive microbiological method to diagnose C. trachomatis [30]. The study was performed in a low-risk population, which increases generalisability to the general population.

Some limitations need to be discussed. As in most prospective cohorts, loss to follow-up might have led towards selection of a more healthy population. The prevalence of $C$. trachomatis infection during pregnancy was higher in those lost to follow-up, which most likely might have led to an underestimation of the observed associations. The low absolute numbers of $C$. trachomatis infection during pregnancy and of specific diagnoses of lower respiratory tract infections might have led to a lack of power to show associations. A previous study did observe a prevalence of $7 \%$ of C. trachomatis in children until age 6 months with respiratory tract infections [31]. However, the study population comprised children presenting with respiratory complaints to a specialised university medical centre, which is not comparable to our general, more healthy study population. Our results might suggest that the risk of overall wheezing is driven by the significant association of $C$. trachomatis with wheezing at age 3 years. However, the effect estimates for wheezing at all other ages are also taken into account and were moderate to strong (effect estimate 1.22-2.06). Their lack of significance was most probably due to a low prevalence of C. trachomatis. The confidence intervals of the percentage change due to mediators were large, which could be due to the large confidence intervals of the original effect estimates. Information on lower respiratory tract infections, wheezing and asthma was obtained by questionnaire, which might have led to reporting bias. For wheezing and asthma, however, validated and frequently used ISAAC questionnaires were used. Last, we did not observe increased risk behaviour, such as multiple sexual partners or treatment for previous sexually transmitted diseases of women with C. trachomatis infection during pregnancy. Although we took various potential confounders and mediators into account, residual confounding might be an issue, as in any observational study. As our study was largely focused on subclinical C. trachomatis infection, we assume the large majority of women were not treated with antibiotics.

\section{Comparison with previous studies}

C. trachomatis infection during pregnancy is associated with neonatal complications such as pre-term birth and neonatal respiratory morbidity $[5,7,8]$. Only one prospective birth cohort study among 8088 
mothers and children demonstrated that treatment for Chlamydia during pregnancy, as reported by the mother as treatment for a gynaecological infection, was associated with a 3-fold increased risk of asthma in the offspring at age 7 years [16]. These women were most likely symptomatic, while due to non-selective urine screening women in our study were most likely asymptomatic. Additionally, in this study treatment for Chlamydia was self-reported and studied in a subanalysis only, which could have led to bias. Our findings suggest that $C$. trachomatis infection during pregnancy is also associated with asthma in later childhood and impaired lung function measurements reflecting airflow limitation and obstruction in the small airways. To the best of our knowledge, the current study is the first to examine the associations of C. trachomatis infection during pregnancy with various types of respiratory morbidity and objective lung function measurements in childhood.

Other studies focused on the associations of chlamydial infections in infancy with childhood outcomes. An observational study compared 18 children hospitalised for chlamydial pneumonia with 19 controls and reported that infants with chlamydial pneumonia were more likely to have asthma at age 7 years, and more often had impaired lung function and bronchial hyperreactivity [14]. Findings from another study comparing 40 children hospitalised for pneumonia or bronchitis in infancy with 71 healthy controls suggested that the association of chlamydial infection in infancy with respiratory diseases is not solely explained by respiratory tract infections [15]. Children with chlamydial lower respiratory tract infections in infancy had an increased risk of cough and were more likely to have an abnormal functional residual capacity until age 5 years than children with non-chlamydial lower respiratory tract infections. Overall, we did not observe associations of $C$. trachomatis infection during pregnancy with lower respiratory tract infections. This might be due to the low prevalence of lower respiratory tract infections in this general, non-hospital-based population. These findings suggest that C. trachomatis infection during pregnancy specifically predisposes children for asthma-related symptoms, but not to general respiratory tract infections.

\section{Explanation and implications of the findings}

Because of the observational design of the study, we cannot draw conclusions on the underlying causality and mechanisms. In vitro studies have elucidated possible direct mechanisms underlying the association between $C$. trachomatis and respiratory health. An in vitro study among neonatal and adult mice showed that mice infected as neonates with Chlamydia muridarum, the murine biovar of $C$. trachomatis, were not able to clear the infection, had C. muridarum-specific IgE in bronchoalveolar fluid and serum, and had an increased production of interleukin (IL)-4 and IL-10, while this was not observed for mice infected in adulthood [32]. Both IgE and IL-4 are of importance in asthma pathogenesis, which might explain our observed association with asthma. Additionally, a related in vitro study demonstrated that alveolar diameter was increased in mice that were infected in the neonatal period, but not in mice infected in infancy or adulthood, which might imply that C. trachomatis directly affects the lungs [33]. These findings suggest that chlamydial infection during early life specifically might lead to developmental lung and immune system adaptations, and consequently an increased risk of wheezing, asthma and lower lung function in later life, but not lower respiratory tract infections. We did not observe any modifying effect of gestational age at diagnosis of C. trachomatis on respiratory health. However, C. trachomatis could have been asymptomatically present for a longer duration of time [34, 35]. C. trachomatis infection during pregnancy may lead to chorioamnionitis $[5,6]$. Chorioamnionitis itself is associated with an increased risk of asthma or impaired lung function especially in children born pre-term [36-39], possibly through immune changes in the child as a result of the chorioamnionitis [40]. In our study, the associations of C. trachomatis infection during pregnancy with school-age lung function and asthma were not explained by pre-term birth, while the association with childhood wheezing was. This might imply that this mediation has only short-term effects. Further studies are needed to assess whether chorioamnionitis explains the observed associations. Lastly, co-infections during pregnancy might play a role in the association of $C$. trachomatis with respiratory health. Previous studies demonstrated that C. trachomatis can co-occur with, for example, Neisseria gonorrhoeae and Trichomonas vaginalis, mostly in high-risk populations, but their association with respiratory health is unclear [41-44].

Our findings are important from both an aetiological and population health perspective. They suggest that a C. trachomatis infection during pregnancy leads to fetal developmental adaptations, which predispose children to asthma-related morbidity, rather through lower lung function than susceptibility for lower respiratory tract infections. Population attributable fractions (calculated as prevalence of exposure among cases $\times(1-1 /$ relative risk)) of C. trachomatis for asthma and overall wheezing in this study were $4.2 \%$ and $1.2 \%$, respectively [45]. We only observed associations of $C$. trachomatis on $\mathrm{FEV}_{1} / \mathrm{FVC}$ and $\mathrm{FEF}_{75 \%}$, and not the other lung function measures. Although the effect sizes are important from an aetiological perspective, they should be carefully considered from a clinical perspective. C. trachomatis infections are 
common and infections are mostly asymptomatic. Screening of pregnant women for C. trachomatis is recommended in the USA for all women under age 25 years or women with risk factors for a sexually transmitted disease [46, 47]. According to local practice, screening for C. trachomatis is not routine practice, although it has been suggested to be cost-effective [48]. C. trachomatis can be treated easily and effectively with a single-dose therapy of azithromycin, even during pregnancy $[46,49]$. Implementation of screening and treatment of pregnant women in the USA has led to a decrease of C. trachomatis infection of the newborn, and morbidity such as pre-term rupture of the membranes and pre-term birth $[47,50]$. Additionally, prevention due to vaccination could be considered in the future [51-53]. Replication of these findings would be of interest, and future studies should focus on the effects of treatment during pregnancy on intra-uterine transmission, chorioamnionitis and long-term offspring health.

\section{Conclusions and future research}

Our results suggest that $C$. trachomatis infection during pregnancy is associated with increased odds of wheezing, asthma and impaired lung function across childhood. The causality of the observed associations and potential underlying biological mechanisms need to be explored.

Acknowledgements: The Generation R Study is conducted by the Erasmus Medical Centre in close collaboration with the School of Law and the Faculty of Social Sciences at the Erasmus University, Rotterdam, the Municipal Health Service, Rotterdam area, and the Stichting Trombosedienst and Artsen Laboratorium Rijnmond (Star-MDC), Rotterdam. We gratefully acknowledge the contribution of children and their parents, general practitioners, hospitals, midwives and pharmacies in Rotterdam.

Author contributions: E.R. van Meel, M. Attanasi, V.W.V. Jaddoe and L. Duijts contributed to the conception and design, acquisition of data, analyses and interpretation of the data, drafted the article, revised it critically for important intellectual content, and gave final approval of the version to be published. I.K.M. Reiss, H.A. Moll and J.C. de Jongste contributed to the conception and design, acquisition of data, revised the drafted manuscript critically for important intellectual content, and gave final approval of the version to be published.

Conflict of interest: None declared.

Support statement: The Generation R Study is made possible by financial support from the Erasmus Medical Centre, Rotterdam, the Erasmus University Rotterdam and the Netherlands Organization for Health Research and Development (ZonMw). V.W.V. Jaddoe received an additional grant from ZonMw (ZonMw-VIDI) and the European Research Council (ERC-2014-CoG-648916). L. Duijts received funding from the European Union's Horizon 2020 co-funded programme ERA-Net on Biomarkers for Nutrition and Health (ERA HDHL) (ALPHABET project (number 696295; 2017); ZonMw (number 529051014; 2017)). The researchers are independent from the funders. The study sponsors had no role in the study design, data collection and analysis, interpretation of data, writing of this report or the decision to submit the article for publication. Funding information for this article has been deposited with the Crossref Funder Registry.

\section{References}

1 Redmond SM, Alexander-Kisslig K, Woodhall SC, et al. Genital Chlamydia prevalence in Europe and non-European high income countries: systematic review and meta-analysis. PLoS One 2015; 10: e0115753.

2 Smith JR, Taylor-Robinson D. Infection due to Chlamydia trachomatis in pregnancy and the newborn. Baillieres Clin Obstet Gynaecol 1993; 7: 237-255.

3 Wilson JS, Honey E, Templeton A, et al. A systematic review of the prevalence of Chlamydia trachomatis among European women. Hum Reprod Update 2002; 8: 385-394.

4 Donders GG, Moerman P, De Wet GH, et al. The association between Chlamydia cervicitis, chorioamnionitis and neonatal complications. Arch Gynecol Obstet 1991; 249: 79-85.

5 Rours GI, de Krijger RR, Ott A, et al. Chlamydia trachomatis and placental inflammation in early preterm delivery. Eur J Epidemiol 2011; 26: 421-428.

6 Rours GI, Duijts L, Moll HA, et al. Chlamydia trachomatis infection during pregnancy associated with preterm delivery: a population-based prospective cohort study. Eur J Epidemiol 2011; 26: 493-502.

7 Silva MJ, Florencio GL, Gabiatti JR, et al. Perinatal morbidity and mortality associated with chlamydial infection: a meta-analysis study. Braz J Infect Dis 2011; 15: 533-539.

8 Olson-Chen C, Balaram K, Hackney DN. Chlamydia trachomatis and adverse pregnancy outcomes: meta-analysis of patients with and without infection. Matern Child Health J 2018; 22: 812-821.

9 Schachter J, Grossman M, Sweet RL, et al. Prospective study of perinatal transmission of Chlamydia trachomatis. JAMA 1986; 255: 3374-3377.

10 Frommell GT, Rothenberg R, Wang S, et al. Chlamydial infection of mothers and their infants. J Pediatr 1979; 95: 28-32.

11 den Dekker HT, Sonnenschein-van der Voort AMM, de Jongste JC, et al. Early growth characteristics and the risk of reduced lung function and asthma: a meta-analysis of 25,000 children. J Allergy Clin Immunol 2016; 137; $1026-1035$

12 Sonnenschein-van der Voort AMM, Arends LR, de Jongste JC, et al. Preterm birth, infant weight gain, and childhood asthma risk: a meta-analysis of 147,000 European children. J Allergy Clin Immunol 2014; 133: 1317-1329.

13 Smith GC, Wood AM, White IR, et al. Neonatal respiratory morbidity at term and the risk of childhood asthma. Arch Dis Child 2004; 89: 956-960. 
14 Weiss SG, Newcomb RW, Beem MO. Pulmonary assessment of children after chlamydial pneumonia of infancy. J Pediatr 1986; 108: 659-664.

15 Harrison HR, Taussig LM, Fulginiti VA. Chlamydia trachomatis and chronic respiratory disease in childhood. Pediatr Infect Dis 1982; 1: 29-33.

16 Xu B, Pekkanen J, Jarvelin MR, et al. Maternal infections in pregnancy and the development of asthma among offspring. Int J Epidemiol 1999; 28: 723-727.

17 Kooijman MN, Kruithof CJ, van Duijn CM, et al. The Generation R Study: design and cohort update 2017. Eur J Epidemiol 2016; 31: 1243-1264.

18 Asher MI, Keil U, Anderson HR, et al. International Study of Asthma and Allergies in Childhood (ISAAC): rationale and methods. Eur Respir J 1995; 8: 483-491.

19 Miller MR, Hankinson J, Brusasco V, et al. Standardisation of spirometry. Eur Respir J 2005; 26: 319-338.

20 den Dekker HT, Jaddoe VWV, Reiss IK, et al. Fetal and infant growth patterns and risk of lower lung function and asthma. The Generation R Study. Am J Respir Crit Care Med 2018; 197: 183-192.

21 Quanjer PH, Stanojevic S, Cole TJ, et al. Multi-ethnic reference values for spirometry for the 3-95-yr age range: the global lung function 2012 equations. Eur Respir J 2012; 40: 1324-1343.

22 Pan W. Akaike's information criterion in generalized estimating equations. Biometrics 2001; 57: 120-125.

23 Harin JW, Hilbe JM. Generalized Estimating Equations. New York, Chapman \& Hall/CRC, 2003.

24 Dick S, Friend A, Dynes K, et al. A systematic review of associations between environmental exposures and development of asthma in children aged up to 9 years. BMJ Open 2014; 4: e006554.

25 Castro-Rodriguez JA, Forno E, Rodriguez-Martinez CE, et al. Risk and protective factors for childhood asthma: what is the evidence? J Allergy Clin Immunol Pract 2016; 4: 1111-1122.

26 Duijts L, Reiss IK, Brusselle G, et al. Early origins of chronic obstructive lung diseases across the life course. Eur J Epidemiol 2014; 29: 871-885.

27 Moraes TJ, Sears MR, Subbarao P. Epidemiology of asthma and influence of ethnicity. Semin Respir Crit Care Med 2018; 39: 3-11.

28 van de Loo KF, van Gelder MM, Roukema J, et al. Prenatal maternal psychological stress and childhood asthma and wheezing: a meta-analysis. Eur Respir J 2016; 47: 133-146.

29 Sterne JA, White IR, Carlin JB, et al. Multiple imputation for missing data in epidemiological and clinical research: potential and pitfalls. BMJ 2009; 338: b2393.

30 Rours GI, Verkooyen RP, Willemse HF, et al. Use of pooled urine samples and automated DNA isolation to achieve improved sensitivity and cost-effectiveness of large-scale testing for Chlamydia trachomatis in pregnant women. J Clin Microbiol 2005; 43: 4684-4690.

31 Rours GI, Hammerschlag MR, Van Doornum GJ, et al. Chlamydia trachomatis respiratory infection in Dutch infants. Arch Dis Child 2009; 94: 705-707.

32 Patel KK, Webley WC. Evidence of infectious asthma phenotype: Chlamydia-induced allergy and pathogen-specific IgE in a neonatal mouse model. PLoS One 2013; 8: e83453.

33 Horvat JC, Starkey MR, Kim RY, et al. Early-life chlamydial lung infection enhances allergic airways disease through age-dependent differences in immunopathology. J Allergy Clin Immunol 2010; 125: 617-625.

34 Sheffield JS, Andrews WW, Klebanoff MA, et al. Spontaneous resolution of asymptomatic Chlamydia trachomatis in pregnancy. Obstet Gynecol 2005; 105: 557-562.

35 Geisler WM. Duration of untreated, uncomplicated Chlamydia trachomatis genital infection and factors associated with chlamydia resolution: a review of human studies. J Infect Dis 2010; 201: Suppl. 2, S104-S113.

36 Kumar R, Yu Y, Story RE, et al. Prematurity, chorioamnionitis, and the development of recurrent wheezing: a prospective birth cohort study. I Allergy Clin Immunol 2008; 121: 878-884.

37 Zhu T, Zhang L, Qu Y, et al. Meta-analysis of antenatal infection and risk of asthma and eczema. Medicine 2016; 95: e4671.

38 McDowell KM, Jobe AH, Fenchel M, et al. Pulmonary morbidity in infancy after exposure to chorioamnionitis in late preterm infants. Ann Am Thorac Soc 2016; 13: 867-876.

39 Getahun D, Strickland D, Zeiger RS, et al. Effect of chorioamnionitis on early childhood asthma. Arch Pediatr Adolesc Med 2010; 164: 187-192.

40 Singh AM, Sherenian MG, Kim KY, et al. Fetal cord blood and tissue immune responses to chronic placental inflammation and chorioamnionitis. Allergy Asthma Clin Immunol 2018; 14: 66.

41 Ginocchio CC, Chapin K, Smith JS, et al. Prevalence of Trichomonas vaginalis and coinfection with Chlamydia trachomatis and Neisseria gonorrhoeae in the United States as determined by the Aptima Trichomonas vaginalis nucleic acid amplification assay. J Clin Microbiol 2012; 50: 2601-2608.

42 Nsuami M, Cammarata CL, Brooks BN, et al. Chlamydia and gonorrhea co-occurrence in a high school population. Sex Transm Dis 2004; 31: 424-427.

43 Carter JE, Whithaus KC. Neonatal respiratory tract involvement by Trichomonas vaginalis: a case report and review of the literature. Am J Trop Med Hyg 2008; 78: 17-19.

44 Weyand NJ. Neisseria models of infection and persistence in the upper respiratory tract. Pathog Dis 2017; 75: $\mathrm{ftx} 031$.

45 Mansournia MA, Altman DG. Population attributable fraction. BMJ 2018; 360: k757.

46 Workowski KA, Bolan GA. Sexually transmitted diseases treatment guidelines, 2015. MMWR Recomm Rep 2015; 64: 1-137.

47 LeFevre ML. Screening for chlamydia and gonorrhea: U.S. Preventive Services Task Force recommendation statement. Ann Intern Med 2014; 161: 902-910.

48 Rours GI, Smith-Norowitz TA, Ditkowsky J, et al. Cost-effectiveness analysis of Chlamydia trachomatis screening in Dutch pregnant women. Pathog Glob Health 2016; 110: 292-302.

49 Adair CD, Gunter M, Stovall TG, et al. Chlamydia in pregnancy: a randomized trial of azithromycin and erythromycin. Obstet Gynecol 1998; 91: 165-168.

50 Angelova M, Kovachev E, Tsankova V, et al. Role and importance of Chlamydia trachomatis in pregnant patients. Open Access Maced J Med Sci 2016; 4: 410-412.

51 Gottlieb SL, Deal CD, Giersing B, et al. The global roadmap for advancing development of vaccines against sexually transmitted infections: update and next steps. Vaccine 2016; 34: 2939-2947. 
52 Phillips S, Quigley BL, Timms P. Seventy years of Chlamydia vaccine research - limitations of the past and directions for the future. Front Microbiol 2019; 10: 70.

53 Abraham S, Juel HB, Bang P, et al. Safety and immunogenicity of the Chlamydia vaccine candidate CTH522 adjuvanted with CAF01 liposomes or aluminium hydroxide: a first-in-human, randomised, double-blind, placebo-controlled, phase 1 trial. Lancet Infect Dis 2019; 19: 1091-1100. 